\title{
PREVALENCIA DE CONDUCTAS ESTEREOTIPADAS Y NO DESEADAS EN EL CABALLO FINO CHILOTE EN LA REGIÓN DE LA ARUCANIA, CHILE.
}

\section{PREVALENCE OF STEREOTYPES AND NON DESIRED BEHAVIORS IN THE FINO CHILOTE HORSE IN THE REGION OF ARUCANIA, CHILE.}

\author{
Christian Tuemmers ${ }^{1,2}$, Angélica Saldivia ${ }^{1,3}$, Carolina Mora ${ }^{1,4}$, \& Javiera Pinto ${ }^{1,5}$ \\ 1- Escuela de Medicina Veteriniaria, Facultad de Recursos Naturales, Universidad \\ Católica de Temuco, Casilla 15-D, Temuco, Chile. \\ 2- Autor para correspondencia, email: ctuemmers@uct.cl \\ 3- email: asaldivia@uct.cl \\ 4- email: cmora@uct.cl \\ 5.- email: jpinto2009@alu.uct.cl
}

Recibido: 22 de mayo de 2017; Aceptado: 20 de junio de 2017.

RESUMEN
El objetivo del presente trabajo fue determinar la prevalencia de conductas estereotipadas y conductas no deseadas en el caballo chilote destinado a hipoterapia en dos centros terapéuticos; CRICAR, escuela de Hipoterapia y Equitación de Carabineros, en el Fundo El Entierro y Fundo Copin; todos ubicados en la novena región de la Araucanía, logrando observar 33 equinos de distinto sexo y edades. Estos fueron analizados en sus distintos hábitats según su rutina de entrenamiento, trabajo y potrero. Las conductas estereotipadas observadas fueron las tres más frecuentes: deambular estereotipado, aerofagia y balanceo del tren posterior, además se registraron conductas anormales: manoteo, morder, lignofagia, comer la cama y cabeceo. Todos estos datos fueron ingresados a la correspondiente planilla Excel donde se analizaron mediante el programa SPSS 20 para obtener los resultados de la estadística descriptiva.

La presentación de estas conductas no deseadas, en los individuos fue relacionada con las condiciones de estabulación ya que no presentan contacto con sus pares, tiempo de alimentación, origen del alimento, restricción física, falta de estimulación, situaciones de estrés las que en sumatoria conducen a un detrimento del bienestar de estos caballos lo que desencadena la expresión de estas conductas.

Respecto de la prevalencias observadas para conductas estereotipadas en caballos destinados a hipoterapia fue $57 \%$ y en el caso de los caballos chilotes baguales fue de un $0 \%$; en el caso de las conductas no deseadas por parte de los caballos que realizan rutinas de hipoterapia se observó $99,6 \%$ y en los caballos baguales $76,7 \%$.

Se observó la presencia de estas conductas en potrillos a pesar de su corta edad y en baguales a pesar de la pobre interferencia del hombre en su hábitat.

Principalmente en el caso de los baguales se cree que las conductas consideradas como anormales en algunos de los casos corresponderían a su comportamiento normal. 
Palabras Claves: Equino, Caballo Fino Chilote, Hipoterapia, Estereotipas, Conductas no deseadas.

\section{SUMMARY}

The aim of the present work was to determine the prevalence of stereotypies conducts and conducts not wished in the chilote horse destined to hipoterapia in two therapeutic centers; CRICAR, school of hippotherapy of Carabineros, also observed wild in the property in the country "El Entierro" and Property in the country "Copin"; all located in the ninth district of the Araucanía, managing observing 33 equine ones of different sex and ages, these they were analyzed in his different habitat according to his routine of training, work and inclusive to herdsman.

The raising of information fulfilled in the lands across a survey the managers of every land compiling information as type of manger, supply, water, upbringing, routine of training and works realized by every individual; in addition the summary of information was effected by means of direct observation by the method of focal sampling by scanning during a time of 24 hours, methodology annotated to described by Tadich and col (2010). The stereotyped observed conducts were of the most frequent three: to stroll stereotyped, aerophagia and balancing of the later train, in addition abnormal conducts were registered: I slap, to bite, crib bitting, to eat the bed and nod. All this information was deposited to the correspondent schedule Excel where they were analyzed through the program SPSS 20 to obtain the results of the descriptive statistics.

The presentation of these conducts not wished in the individuals was related to the conditions of stabling since they do not present contact with his couples, time of supply, origin of the food, physical restriction, lacking in stimulation, situations of stress those that in add up to lead to a detriment of the well-being of these horses what unleashes the expression of these conducts. The statistical analysis I realize by means of the test chi-squared, who considers a result to be significant $p \leq 0,05$ and not significantly a value $\mathrm{p} \geq 0,05$.

Respect of the prevalence's observed for conducts stereotyped in horses destined to hippotherapy was $57 \%$ and in case of the horses chilotes wild it was of $0 \%$; in case of the conducts not wished on the part of the horses that realize routines of hippotherapy I observe \% and the wild horses \%

I observe the presence of these conducts in foals in spite of his short age and in wild in spite of the poor interference of the man in his habitat. Principally in case of the wild ones one believes that the conducts considered since abnormal in some of the cases they would correspond to his normal behavior.

Keywords: Equine, Fino Chilote Horse, hippotherapy, stereotypies, unwanted behaviors.

\section{Introducción}

El caballo Fino Chilote o Mampato como también es conocido, se considera un recurso zoogenético valioso ya que posee cualidades únicas en el mundo que la hacen ser reconocida como raza oficialmente a nivel internacional desde el año 1999. Al ser una especie introducida por los españoles entre los siglos XVI y XVII en el archipiélago de 
Chiloé, su origen se asocia al caballo Asturcón y Andaluz de la Península Ibérica y le proporcionan características como su rusticidad, mansedumbre, exiguo tamaño e importante capacidad de carga (Escobar y col 1998).

Debido a la incorporación de nuevas razas equinas a la Isla de Chiloé; esta emblemática raza se vio afectada incluso bordeando la extinción; motivo por el cual fue introducida en un programa de recuperación, conservación y caracterización de la raza llevada a cabo por el Instituto de Investigaciones Agropecuarias (INIA) y la Universidad Austral de Chile (UACh), proyecto financiado por la Fundación de Innovación Agraria y la Secretaria Ministerial de Agricultura. A partir de este programa se realizó una clasificación en base a grupos sanguíneos y marcadores moleculares como secuencias repetidas (SSR) o Microsatélites, utilizados para análisis de paternidad con el objetivo de construir un registro genealógico.

Esta raza se utiliza para transporte, mascota, turismo ecuestre, trabajo liviano, pero principalmente estos últimos años como ayudante terapeuta de hipoterapia. Es así que el manejo humano ha generado alteraciones fisiológicas y psicológicas de estos ejemplares; lo que ha generado comportamientos extraños y poco habituales que son reconocidos como anormales. Estos caballos están adaptados a la vida salvaje pero debido a la intervención del hombre en su intento por domesticarlo lo ha sometido a situaciones de estrés como lo son la estabulación permanente, trabajo de carga, acceso restringido de alimento y agua conllevando a que el individuo presente conductas anormales que no presentan una función definida que incluso pueden ocasionar daños en el animal. Esta raza de caballos única en el mundo es un recurso invaluable desde el punto de vista de sus antecedentes históricos, caracteres morfológicos (Voeltz, 1996), al igual que sus caracteres antigénicos (Barrera, 1998), los cuales rectifican su origen en la península Ibérica.

Muñoz y col (2009) describen dos grupos de comportamientos anormales; las conductas anormales y las estereotipadas o estereotipias. En el caso de las primeras corresponden a un acto aberrante con un fin determinado; mientras que en el caso de las segundas son aberrantes, repetitivas, se caracterizan por manifestar una secuencia de movimientos recurrentes e invariables, sostenidos y que el individuo tiene dificultad para detenerlos, además no presentan un propósito o función definida.

El objetivo de este estudio fue determinar la frecuencia de presentación de conductas estereotipadas $u$ anormales en el Caballo Chilote de Hipoterapia y bagual no descritas, por lo que es de suma relevancia conocer la prevalencia de estas para así determinar su origen y al mismo tiempo analizar la etología de esta raza frente a los distintos escenarios en los cuales habita y trabaja. De esta manera se permitirá contar con registros necesarios para prevenir la presentación de estas conductas. Además, el contar con un estudio descriptivo nos orientará para visualizar nuestras falencias en el bienestar animal, ya que estas conductas generan repercusiones en el ámbito productivo ya que limitan el desempeño, rendimiento y comportamiento.

La hipótesis que se planteó es que debido a la domesticación de esta raza autóctona y bagual sumado a los manejos a que es sometida debiesen presentar conductas anormales y estereotipias.

Material Biológico:

MATERIALES Y MÉTODOS 
Se observaron 33 ejemplares de la raza caballar Fino Chilote en la región de la Araucanía; estos equinos eran de distinto sexo, siendo 19 hembras y 14 machos, con edades desde potrillos de año y medio hasta los 18 años, destinados a hipoterapia y baguales, los primeros se encontraban en el CRICAR: Centro de rehabilitación de Carabineros, ubicado en la Escuela de Carabineros Alguacil Mayor Juan Gómez de Almagro, sector las Quilas, también se visitó un centro de equitación e hipoterapia en la localidad de Padre las Casas. Respecto de los baguales se visitó el Fundo "El Entierro" situado en Caivico y finalmente en la localidad de Curacautin se encuentra emplazado el Fundo "Copin".

Material de registro:

- Pauta de Registro

- Cámara digital

Métodos:

La metodología de visita fue durante el día y la noche para cumplir con las 24 horas de observación e inclusive en algunos recintos se repitió la visita para cerciorar que las conductas observadas no eran producto de la interpretación del observador o gatilladas por alguna circunstancia propia del entrenamiento u hábitat. Antes de cualquier intervención se realizó una encuesta a los encargados o propietarios del recinto para recopilar antecedentes como sexo, edad, inicio entrenamiento según el caso, alimentación, características de la pesebrera, presencia de estereotipa o conducta anormal entre otras. Durante las 24 hrs de observación los caballos destinados a hipoterapia permanecían alrededor de 19 hrs estabulados, en el caso de los baguales solo estaban a potrero y se debía privilegiar la observación diurna ya que las fechas en las cuales se observaron había presencia de neblina e inclusive noches muy oscuras y el observar con luz artificial podría ocasionar una alteración en su comportamiento evidentemente.

En los centros de hipoterapia se observaron los individuos antes de recibir su primera ración de alimento a diferencia de los baguales que cumplían a cabalidad e incluso superaban de manera intermitente las 16 hrs de forrajeo al día. Luego se comenzó con un registro de las conductas a través de observación directa durante 24 horas continuas, finalizando después de la primera ración de alimento. Cada individuo fue observado 5 minutos donde luego se realizaba un barrido general cada 30 minutos en el caso de los estabulados, idéntica metodología se utilizó en los baguales pero fue una observación dirigida a una manada ya que en ambos predios existían dos manadas. En las pesebreras donde se identificaron los individuos con estereotipas o conductas no deseadas e inclusive detectadas in situ, se instaló una cámara digital con grabación continua de 5 a 10 minutos.

Registro de Estereotipias y conductas no deseadas:

Según Navarrete y col (2015) en un estudio realizado se analizaron las implicancias de las relaciones entre algunos factores y la presentación de las estereotipias y conductas no deseadas, clasificándose de la siguiente manera (Cuadro 1); esta referencia se uso 
para determinar las conductas prevalentes, siendo registrados en el anexo 2 y $3,3 \mathrm{~A}, 3$ B, y 3 C.

Cuadro 1.- Etograma conductas observadas, clasificación en estereotipadas o conductas no deseadas, descripción y origen oral o locomotor.

\begin{tabular}{|c|c|c|c|}
\hline & Conducta & Oral/Locomotor & Descripción \\
\hline 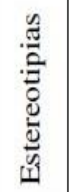 & $\begin{array}{l}\text { Aerofagia } \\
\text { Balanceo estereotipado } \\
\text { Caminata estereotipada }\end{array}$ & $\begin{array}{l}\text { Oral } \\
\text { Locomotor } \\
\text { Locomotor }\end{array}$ & $\begin{array}{l}\text { Ingreso de aire hacia el esófago con o sin apoyo de los incisivos. } \\
\text { Movimiento de balanceo lateral del tren anterior. } \\
\text { Caminar repetidamente dentro de la pesebrera }\end{array}$ \\
\hline 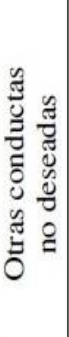 & $\begin{array}{l}\text { Lignofagia } \\
\text { Comer cama } \\
\text { Otras conductas orales } \\
\text { Cabeceo } \\
\text { Agresividad }\end{array}$ & $\begin{array}{l}\text { Oral } \\
\text { Oral } \\
\text { Oral } \\
\text { Locomotor } \\
\text { Locomotor }\end{array}$ & $\begin{array}{l}\text { Ingestión de madera. } \\
\text { Ingestión del material de cama. } \\
\text { Lamer o morder objetos sin fin nutricional. } \\
\text { Movimientos verticales repetidos de la cabeza. } \\
\text { Amenazas de o realización de mordidas o patadas dirigidas a humanos. }\end{array}$ \\
\hline
\end{tabular}

Análisis estadístico:

La información recopilada fue ingresada a una planilla del programa Excel con el objetivo de resumir los datos (Tabla 1 y 2 ) respecto de las estereotipas y las conductas no deseadas (Tabla 3 y 4 ).

Para la obtención de porcentajes de estadística descriptiva los datos antes mencionados fueron ingresados al programa SPSS 2.0, dando como resultado la presencia de estereotipias y conductas no deseadas en el Caballo Fino Chilote.

Con este mismo programa se realizaron análisis de tablas de contingencia respecto de las conductas no deseadas en caballos de hipoterapia como baguales. A partir de estas tablas de contingencia de doble entrada se realizó el análisis estadístico Chi cuadrado, dando grados de significancia a $P \leq 0,05$ y no significativo a un $P \geq 0,05$.

Tabla 1. Caballos destinados a hipoterapia.

\begin{tabular}{lll}
\hline Centro & Estereotipa & Conducta no deseada \\
\hline CRICAR & Aerofagia s/ fijación & Comer cama \\
& Deambular estereotipado & Morder \\
& Lignofagia \\
\hline
\end{tabular}




\begin{tabular}{|c|c|c|}
\hline & Balanceo tren anterior & $\begin{array}{l}\text { Cabeceo } \\
\text { Manoteo }\end{array}$ \\
\hline Ernaldo Bustamante & & $\begin{array}{l}\text { Comer Cama } \\
\text { Lignofagia }\end{array}$ \\
\hline
\end{tabular}

Tabla 2. Caballos Baguales.

\begin{tabular}{|c|c|c|}
\hline Fundo & Estereotipa & Conducta no deseada \\
\hline \multirow[t]{4}{*}{ El Entierro } & & Acicalamiento \\
\hline & & Manoteo \\
\hline & & Morder \\
\hline & & Cabeceo \\
\hline \multirow[t]{3}{*}{ Copin } & & Acicalamiento \\
\hline & & Manoteo \\
\hline & & Cabeceo \\
\hline
\end{tabular}

Tabla 3 Conductas no deseadas equinos hipoterapia $\mathrm{N}=7$

\begin{tabular}{lcc}
\hline Conducta no deseada & $\mathrm{N}^{\circ}$ conducta no deseada & Porcentaje \\
\hline Manoteo & 1 & $14,2 \%$ \\
Morder & 1 & $14,2 \%$ \\
Lignofagia & 3 & $42,8 \%$ \\
Comer cama & 1 & $14,2 \%$ \\
Cabeceo & 1 & $14,2 \%$ \\
Acicalamiento & 0 & $0 \%$ \\
Presentación & 7 & $99,6 \%$ \\
No presenta & & $0.4 \%$ \\
Total & & $100 \%$ \\
\hline
\end{tabular}

Tabla 4 Conductas no deseadas en baguales $\mathrm{N}=26$

\begin{tabular}{lcc}
\hline Conducta no deseada & $\mathrm{N}^{\circ}$ conducta no deseada & Porcentaje \\
\hline Manoteo & 7 & $26,9 \%$ \\
Morder & 2 & $7,6 \%$ \\
Lignofagia & 0 & 0 \\
Comer cama & 0 & 0 \\
Cabeceo & 2 & $7,6 \%$ \\
Acicalamiento & 9 & $34,6 \%$ \\
Presentación & 20 & $76,7 \%$ \\
No presenta & & $24,3 \%$ \\
Total & & $100 \%$ \\
\hline
\end{tabular}

Posteriormente se realizó en cada conducta no deseada la correlación de cada variable para ver si se relacionan de igual forma las conductas deseadas en los equinos con diferente manejo, mediante el método de análisis cualitativo de datos.

5. RESULTADOS. 
De una población total de 33 caballos Fino Chilote de los cuales 7 eran destinados a trabajo de hipoterapia y los 26 restantes eran baguales; solo los equinos destinados a hipoterapia presentaron conductas estereotipias, dentro de este grupo cuatro de ellos presentaron alguna conducta estereotipada perteneciendo al centro de hipoterapia CRICAR, obteniendo como resultado para la aerofagia $14,2 \%$, en el caso de deambular estereotipado $28,5 \%$ y para el balanceo del tren anterior $14,2 \%$, porcentajes que representan una prevalencia del $56,9 \%$ de la población destinada a hipoterapia (tabla 5 y 6$)$.

Tabla 5 estereotipa en caballos de hipoterapia. $\mathrm{N}^{\circ}=7$

\begin{tabular}{lcl}
\hline Estereotipa & Numero de estereotipia & Porcentaje \\
\hline Aerofagia & 1 & $14,2 \%$ \\
Deambular estereotipado & 2 & $28,5 \%$ \\
Balanceo del tren anterior & 1 & $14,2 \%$ \\
Total & 4 & $56,9 \%$ \\
No Presenta & 3 & $43,1 \%$ \\
\hline
\end{tabular}

Tabla 6 estereotipa en baguales $\mathrm{N}^{\circ} 26$

\begin{tabular}{lcl}
\hline Estereotipa & Numero de estereotipia & Porcentaje \\
\hline Aerofagia & 0 & $0 \%$ \\
Deambular estereotipado & 0 & $0 \%$ \\
Balanceo del tren anterior & 0 & $0 \%$ \\
Total & 0 & $0 \%$ \\
No Presenta & 26 & $100 \%$ \\
\hline
\end{tabular}

Para el caso de las conductas no deseadas observadas en los dos grupos de la población los datos obtenidos en los equinos destinados a Hipoterapia son para el caso de manoteo $14,2 \%$, para morder $14,2 \%$, para lignofagia un $42,8 \%$, comer cama un $14,2 \%$, cabeceo $14,2 \%$ y en el caso del acicalamiento no se presentó la conducta. Respecto de los caballos que habitan en condiciones naturales que corresponde a un numero de 26 individuos en estos también existió la presentación de conductas no deseadas un poco más elevados en algunas conductas los porcentajes respecto de los otros individuos siendo en el caso del manoteo $26,9 \%$, para morder $7,6 \%$, lignofagia y comer cama no se presentaron en estos individuos, para la conducta de acicalamiento fue de $34,6 \%$, lo que resulta en una presentación de $76,7 \%$, sobre las conductas no deseadas.

Durante las 24 horas de observación la presentación de conductas estereotipadas en el centro de hipoterapia de Carabineros de Chile, fueron alrededor de las 18:30 hrs posteriores a recibir la última comida, quedar encerrados, sin fuente de luz artificial ni contacto visual con los otros individuos.

Los caballos pertenecientes al Centro de Hipoterapia de Padre Las Casas presentaron solo conductas no deseadas en el transcurso del día, inclusive en el patio de entrenamiento y por sobre todo en sus pesebreras al pasar casi $16 \mathrm{hrs}$ encerrados solo teniendo contacto entre ellos en una pesebrera doble pero con separaciones.

Luego de tabulados los datos en las tablas ya presentadas y los anexos $1,2,3,3 \mathrm{~A}, 3 \mathrm{~B}$ y 3 C. Se procedió a realizar el análisis cualitativo para cada presentación de conductas 
no deseadas en cada grupo de individuos mediante una tabla de contingencia de doble entrada evaluando el grado de significancia para cada conducta no deseada mediante Chi- cuadrado. Obteniendo como resultado lo descrito en la tabla 7 , lo cual indica que no existe correlación entre las variables analizadas.

Tabla 7. Significancia de las variables analizadas.

\begin{tabular}{lll}
\hline Variable & $\mathrm{P}$ & Significancia \\
\hline CND/Hipoterapia & $\geq 0,05$ & No \\
CND/Baguales & $\geq 0,05$ & No \\
\hline
\end{tabular}

En el CRICAR la alimentación de los individuos es en base a fardo de alfalfa fraccionado en tres entregas al día, 7:00, 12:00 y 17:00 hrs, en conjunto a la alfalfa se lo proporciona una ración de avena a medio día, y la disponibilidad de agua es ad libitum.

En el Centro de Hipoterapia de Padre Las Casas los caballos reciben diariamente $1.5 \mathrm{~kg}$ de avena y pasto de ballica avena en promedio unos $3 \mathrm{~kg}$. dosificados durante el día según su entrenamiento o si son sacados a potrero previo al entrenamiento y su consumo de agua es ad libitum.

En el caso de los caballos mantenidos bajo condiciones naturales, estos se alimentan de pradera natural, agua de estero o riachuelo que atraviesa el predio e inclusive se les observo comer rosa mosqueta y arbustos.

\section{Discusión}

El objetivo de este trabajo de investigación como ya se mencionó anteriormente fue determinar la presentación de conductas estereotipadas y conductas anormales en la raza caballar Fino Chilote tanto destinados a Hipoterapia como en los individuos que habitan libremente en los campos de la novena región de la Araucanía en una población total de 33 equinos con una edad de 2 a 18 años y de diferentes sexos.

En base a este objetivo y levantamiento de información en base al método de muestreo focal continuo durante 24 hrs, metodología similar a la descrita por Tadich (2010) en su estudio preliminar de sementales de la misma raza; la presentación de estereotipias solo fue manifestada en los individuos que son sometidos a un entrenamiento, manejo, encierro, con un estado de bienestar animal deficiente los que se agrupan en caballos de Hipoterapia, presentando una prevalencia de 56,9\% donde este porcentaje, está compuesto por la presentación de 1 equino con aerofagia que representa el 14,2\%, 2 con deambular estereotipado siendo un $28,5 \%$ y 1 individuo con deambular estereotipado equivalente al 14,2\%; estos porcentajes resultan muy elevados a simple vista versus los descritos por otros autores en otras razas como Tadich y col (2012) quien describe una prevalencia total de $3,1 \%$ en caballos Criollos Chilenos. Otro factor a considerar en los porcentajes de presentación de conductas estereotipadas es que la población de caballos que se dedican a hipoterapia es pequeña, siendo solo 7 individuos. Lamentablemente desde el punto de vista comparativo no existen descripciones en esta raza sobre las conductas estereotipadas en sus diferentes hábitats.

Además en este estudio los equinos que se encontraban a potrero en su estado natural propio de los equinos, con una jerarquización, enfrentado a condiciones de búsqueda de 
alimento, juegos de grupo, sin competencia por el alimento ni estrés desde el punto de vista de algún manejo de bienestar; la presentación de conductas no deseadas de cualquier tipo (cuadro 1 ) en los baguales fue de $76,7 \%$ y en los caballos destinados a hipoterapia 99,6\%, valores porcentuales muy diferentes a los descritos por Navarrete y col (2015) a pesar del tamaño mayor de la población.

Las diferencias observadas a nivel porcentual en la presentación de estereotipias en los equinos de hipoterapia puede deberse a diversos factores; ya que en ambos centros de hipoterapia, el entrenamiento es súper riguroso en cada individuo, no existe contacto visual entre ellos cuando se encuentran en las pesebreras, se encuentran en galpones que tienen otras razas de equinos con distintos fines, la alimentación es súper restringida y de mediana calidad al igual que el tiempo para compartir en manada como es el comportamiento normal de los equinos (Márquez, 2010).

Como concluyen McBride y Hemmings (2009) que el estrés crónico es la causa desencadenante para la presentación de alteraciones conductuales, relacionadas directamente con el hecho de no cubrir las necesidades específicas mínimas de bienestar animal para la especie equina en condiciones de estabulación.

Mason(1991) describe que la presentación de una conducta no deseada u estereotipia depende de la especie, haciendo incapie que dentro de cada especie pueden existir variaciones entre individuos, referente a flexibilidad y repetitividad del patrón conductual; lo cual queda en evidencia en este estudio, ya que en la misma población se observaron diferencias en los patrones conductuales de las estereotipias y las conductas no deseadas a pesar de que los grupos presentaban condiciones similares de entrenamiento, alimentación y hábitat.

Los entrenadores y propietarios consideran un problema a los caballos que presentan alteraciones conductuales, las que a su vez son confundidas con su patrón de comportamiento normal desconocido por estos; estas conductas consideradas anormales deben ser evaluadas e interpretadas bajo el escenario en que los equinos realizan su vida naturalmente, por lo que con el fin de prevenir o mejorar esta observación se deben proporcionar a los individuos una estabulación similar en lo ideal a la vida natural, aumentar la cantidad de fibra en la ración, mejorar el contacto entre individuos y de esta manera reducir el estrés.

\section{Conclusión}

De acuerdo a la información recolectada y analizada respecto de la población de caballos Fino Chilote en diferentes condiciones de hábitat y manejos, se concluye que, la prevalencia de conductas estereotipadas en los caballos chilotes corresponde a un $56,9 \%$, siendo la más prevalente de las tres descritas, el deambular estereotipado con un $28,5 \%$, observando solo estas conductas en los caballos destinados a hipoterapia, claramente por el tiempo de estabulación al que son sometidos, alimentación, adiestramiento prematuro, rutina de trabajo, personal que interactúa con ellos, consumando de esta manera un grado de bienestar animal ínfimo, propiciando de esta manera las condiciones óptimas para su percutor y consumación; muy distinto a lo observado en los caballos que habitan libres en los campos con mínima intervención del hombre, en este grupo mayor de animales tuvieron $0 \%$ de presentaciones estereotipadas, confirmando solo porcentualmente que las condiciones de origen social, 
bienestar o patológicas que gatillan las estereotipias tienen directa relación con su presentación.

Respecto de las conductas no deseadas en los caballos fino chilote bajo lo descrito por la literatura y las observaciones obtenidas en terreno por varias horas; estas conductas corresponderían al comportamiento propio de la raza que no ha sido tan estudiada a diferencia de otros ejemplares equinos; estos caballos al realizar estas conductas se apreciaban de cierta manera relajados y sin ninguna situación que gatillara la manifestación de alguna conducta.

La asociación entre la condición de ser un caballo destinado a hipoterapia fue de correlación positiva para la presentación de estereotipias como de conductas no deseadas.

\section{REFERENCIAS}

Barrera M, 1998. Parámetros morfológicos y tipificación de polimorfismos antigénicos eritrocitarios y bioquímicos como base del stud book de la raza caballo chilote, Tesis, Medicina Veterinaria Universidad Austral de Chile, Facultad de Ciencias Veterinarias, Valdivia, Chile.

Escobar A, J Oltra, M Ortiz, J Voeltz, 1998. Caballo Chilote, FAO Animal Genetic Resources Information Vol. 23, 41-47.

Márquez C, A Escobar, T Tadich, 2010. Características de manejo y conducta en caballos estabulados en el sur de Chile: Estudio preliminar, Archivos Medicina Veterinaria, Vol. 42, 203 - 207.

Mason G. 1991. Stereotypies - a critical review. Anim Behav 41, 1015-1037.

Mason G, J Rushen, 2006. A decade-or-more's progress in understanding stereotypic behavior. In: Mason, G., Rushen, J. (Eds.), Stereotypic Animal Behavior: Fundamentals and Applications to Welfare. CAB International, Wallingford, UK, 1 - 18.

McBride S, A Hemmings, 2005. Altered mesoaccumbens and nigro-striatal dopamine physiology is associated with stereotypy development in a non-rodent species. Behav. Brain. Res. 159, 113-118.

Navarrete D, C Hamilton-West, N Stephens, C Weber, T Tadich, 2015. Factores de riesgo para la presentación de conductas no deseadas en equinos de deporte en Chile, Archivo Medicina Veterinaria, 47, 77-84

Tadich T, O Araya, 2010. Conductas no deseadas en equinos, Archivos Medicina Veterinaria, Vol. 42, 29-41.

Tadich T, J Smulders, O Araya, C Nicol, 2012. Husbandry practices associated with the presentation of abnormal behaviours in Chilean Creole horses. Arch. Med. Vet. $44,279-284$. 
Sustainability, Agri, Food and Environmental Research, 5(2), 2017: 1-11

Voeltz J, 1996, Descripción morfológica del Caballo Chilote y su distribución en la isla de Chiloé, Tesis, Medicina Veterinaria Universidad Austral de Chile, Facultad de Ciencias Veterinarias, Valdivia, Chile. 